\title{
Bevorzugte Report Items für systematische Übersichten und Meta-Analysen: Das PRISMA-Statement
}

Wörtliche Übersetzung aus dem Englischen

\author{
A. Ziegler ${ }^{1}$ \\ G. Antes ${ }^{2}$ \\ I. R. König1 \\ ${ }^{1}$ Institut für Medizinische Biometrie und \\ Statistik, Universität zu Lübeck, Universitäts- \\ klinikum Schleswig-Holstein, Campus \\ Lübeck, Lübeck \\ Deutsches Cochrane Zentrum, Freiburg
}

\section{Einleitung}

$\nabla$

Systematische Übersichten und Meta-Analysen sind in der medizinischen Versorgung immer wichtiger geworden. Kliniker halten sich damit in ihrem Arbeitsgebiet auf dem Laufenden $[28,32]$. Außerdem werden sie oft als Ausgangspunkt für die Entwicklung von klinischen Leitlinien eingesetzt. Drittmittelgeber fordern möglicherweise systematische Übersichten als Rechtfertigung für weitere Forschungsvorhaben [7], und auch einige medizinische Fachzeitschriften entwickeln sich in diese Richtung [42]. Der Nutzen einer systematischen Übersichtsarbeit hängt wie bei jeder Forschung davon ab, was gemacht wurde, was die Ergebnisse waren, und wie klar der Bericht ist. Da die Qualität der Reports systematischer Übersichten ebenso wie bei anderen Publikationen variiert, können Leser die Stärken und Schwächen dieser Arbeiten nicht immer vollständig erfassen.

Die Qualität von Reports zu Übersichtsarbeiten wurde in verschiedenen Studien bewertet. So untersuchte Mulrow 198750 Übersichtsarbeiten, die in vier führenden Medizinischen Fachzeitschriften 1985 und 1986 veröffentlicht wurden [27]. Keine der Arbeiten erfüllte alle 8 wissenschaftlichen Kriterien, wie z.B. die Beurteilung der Güte der eingeschlossenen Studien [27]. Im Jahr 1987 untersuchen Sacks et al. [30] die Angemessenheit des Reports von 83 Meta-Analysen unter Verwendung von 23 Güteeigenschaften aus 6 Bereichen. Die Reports waren allgemein mangelhaft; zwischen 1 und 14 Eigenschaften waren adäquat beschrieben (Mittelwert 7,7; Standardabweichung 2,7). Nur wenig Verbesserung war bei einer Aktualisierung dieser Studie im Jahr 1996 erkennbar [31].

Um den suboptimalen Berichten von Meta-Analysen entgegenzuwirken, entwickelte eine internationale Arbeitsgruppe 1996 als Leitfaden das QUOROM Statement („QUality Of Reporting Of
Meta-Analyses,“ Qualität des Reports von MetaAnalysen) [23]. Schwerpunkt dieses Leitfadens war das Berichten von Meta-Analysen randomisierter kontrollierter Studien. In dieser Arbeit wird eine Überarbeitung dieses Leitfadens beschrieben. Die überarbeitete Empfehlung wird PRISMA („Preferred Reporting Items for Systematic reviews and Meta-Analyses“, Bevorzugte Report Items für systematische Übersichten und Meta-Analysen) genannt, um eine Reihe konzeptueller und praktischer Fortschritte bei systematischen Übersichten zu adressieren (Box 1).

\section{Terminologie $\nabla$}

Die Terminologie, die zur Beschreibung einer systematischen Übersichtsarbeit und Meta-Analyse verwendet wird, hat sich im Laufe der Zeit entwickelt. Ein Grund für die Änderung des Namens von QUOROM in PRISMA ist, dass der Begriff sowohl systematische Übersichten als auch Meta-Analysen beinhalten sollte. Wir haben die Definitionen übernommen, die von der Cochrane Collaboration verwendet werden [15]. Eine systematische Übersicht ist eine Übersicht zu einer klar formulierten Fragestellung, die systematisch und mit spezifischen Methoden relevante Forschungsergebnisse identifiziert, auswählt und kritisch beurteilt. Daten der eingeschlossenen Studien werden gesammelt und analysiert. Statistische Verfahren (Meta-Analyse) können benutzt werden, um die Ergebnisse der eingeschlossenen Studien zu analysieren und zusammenzufassen. Meta-Analyse bezieht sich auf die Verwendung statistischer Verfahren in einer systematischen Übersicht, um die Ergebnisse der eingeschlossenen Studien zusammenzufassen.

\section{Entwicklung des PRISMA -Statements}

Ein dreitägiges Treffen mit 29 Teilnehmerinnen und Teilnehmern fand in Ottawa, Kanada, im Juni 2005 statt. Die Gruppe bestand aus Autoren von Übersichten, Methodikern, Klinikern, medizinischen Herausgebern und einem Endnutzer. Das Ziel des Treffens in Ottawa war die Überarbeitung und Erweiterung der QUOROM-Checkliste und des Flussdiagramms.

Vor dem Treffen erstellte der Vorstand eine systematische Übersicht zu Studien, die die Qualität des Reports von systematischen Übersichten berichteten, sowie eine umfassende Literaturrecherche zum Identifizieren methodischer und
D. Moher 1,2

A. Liberati 3,4

J. Tetzlaff ${ }^{1}$

D. G. Altman ${ }^{5}$

Schlüsselwörter

Evidenzbasierte Anwendung

Meta-Analyse

Publikationsstandard

Qualitätskontrolle

Systematische Übersicht

Keywords

evidence-based practice

meta-analysis

publishing standards

quality contro

systematic reviews

Institut

${ }^{1}$ Ottawa Methods Centre, Ottawa Hospital Research Institute, Ottawa, Ontario, Kanada 2Department of Epidemiology and Community Medicine, Faculty of Medicine, University of Ottawa, Ottawa, Ontario, Kanada 3 Università di Modena e Reggio Emilia, Modena, Italien

${ }^{4}$ Centro Cochrane Italiano, Istituto Ricerche Farmacologiche Mario Negri, Mailand, Italien ${ }^{5}$ Centre for Statistics in Medicine, University of Oxford, Oxford, Großbritannien

Bibliografie

DOI $10.1055 / s-0031-1272978$ Dtsch Med Wochenschr 2011 136: e9-e15 - (c) Georg Thieme Verlag KG Stuttgart · New York . ISSN 0012-0472

Korrespondenz Univ.-Prof. Dr. Andreas Ziegler Institut für Medizinische Biometrie und Statistik Universität zu Lübeck Universitätsklinikum SchleswigHolstein, Campus Lübeck, Maria-Goeppert-Str. 1

23562 Lübeck

Tel. 0451/500 2789

Fax 0451/500 2999

eMail

ziegler@imbs.uni-luebeck.de 
Tab. 1 Checkliste zum Bericht einer systematischen Übersicht oder einer Meta-Analyse.

\begin{tabular}{|c|c|c|}
\hline $\begin{array}{l}\text { Publikations- } \\
\text { abschnitt }\end{array}$ & & Zusammenfassung \\
\hline \multicolumn{3}{|l|}{ Titel } \\
\hline Titel & 1 & Identifikation als Bericht einer systematischen Übersicht, Meta-Analyse oder beidem. \\
\hline \multicolumn{3}{|l|}{ Zusammenfassung } \\
\hline $\begin{array}{l}\text { Strukturierte } \\
\text { Zusammenfassung }\end{array}$ & 2 & $\begin{array}{l}\text { Strukturierte Zusammenfassung mit den Stichworten (sofern geeignet): Hintergrund; Ziele; Datenquellen; Auswahl- } \\
\text { kriterien der Studien, Teilnehmer und Interventionen; Bewertung der Studie und Methoden der Synthese; Ergebnisse; } \\
\text { Einschränkungen; Schlussfolgerungen und Implikation der wichtigsten Ergebnisse; Registrierungsnummer der } \\
\text { systematischen Übersicht. }\end{array}$ \\
\hline
\end{tabular}

Einleitung

Hintergrund und

Ziele

\section{Methoden}

Protokoll und

Registrierung

Auswahlkriterien

Informations

quellen

Suche

Auswahl der

Studien

Prozess der Daten-

gewinnung

Datendetails

Wissenschaftlicher Hintergrund und Begründung der Studie.

Präzise Angabe der Fragestellungen mit Bezug auf Teilnehmer, Interventionen, Vergleiche, Zielkriterien und Studiendesign (engl.: participants, interventions, comparisons, outcomes, and study design; PICOS)).

$5 \quad$ Existiert ein Studienprotokoll für die Übersichtsarbeit? Wenn ja, wo kann es gefunden bzw. wie kann es bezogen werden (z.B. Webseite); wenn verfügbar: Informationen zur Registrierung einschließlich Angabe der Registrierungsnummer.

6 Merkmale der Studien (z. B., PICOS, Dauer der Nachbeobachtung) und der Berichte (z. B., Zeitraum der Studien, Sprache, Publikationsstatus), die als Auswahlkriterien verwendet wurden, mit Begründung.

7 Beschreibung aller Informationsquellen (z. B., Datenbanken mit Zeitpunkten der Berichterstattung, Kontakt mit Autoren von Studien, um zusätzliche Studien zu identifizieren), die bei der Suche verwendet wurden einschließlich des letzten Suchdatums.

8 Beschreibung der vollständigen elektronischen Suchstrategie für mindestens eine Datenbank, einschließlich gewählter Limitierungen, so dass die Suche repliziert werden könnte.

9 Beschreibung des Auswahlprozesses von Studien (das heißt Vorauswahl, Eignung, Einschluss in die systematische Übersicht und, falls zutreffend, in die Meta-Analyse).

10 Beschreibung der Methode der Datenextraktion aus Berichten (z. B. Erhebungsbogen, unabhängig, doppelt) und alle Prozesse, um Daten von Untersuchern zu erhalten und zu bestätigen.

11 Aufzählung und Definition aller Variablen, nach denen gesucht wurde (z. B., PICOS, Finanzierungsquellen) sowie Annahmen und durchgeführte Vereinfachungen.

Risiko der Verzerrung in

den einzelnen

Studien

Effektschätzer

Synthese der

Ergebnisse

Risiko der Verzerrung

über Studien hinweg

Zusätzliche Analysen

Methoden zur Beurteilung des Risikos von Verzerrungen der einzelnen Studien (einschließlich der Angabe, ob dieses auf der Studienebene oder für das Zielkriterium durchgeführt wurde) und wie diese Information bei der Datensynthese berücksichtigt wurde.

13 Wichtigste Effektschätzer (z. B. relatives Risiko, Mittelwertsdifferenz).

14 Beschreibung der Methoden zum Umgang mit den Daten und der Kombination der Ergebnisse der Studien; falls diese berechnet wurden, einschließlich Maßzahlen zur Homogenität der Ergebnisse (z. B. I2) für jede Meta-Analyse.

15 Beschreibung der Beurteilung des Risikos von Verzerrungen, die die kumulative Evidenz beeinflussen könnten (z. B. Publikationsverzerrung, selektives Berichten innerhalb von Studien).

16 Methoden für zusätzliche Analysen (z.B. Sensitivitätsanalysen, Subgruppenanalysen, Meta-Regression) mit Beschreibung, welche vorab spezifiziert waren.

Ergebnisse

Auswahl der Studien

Anzahl der Studien, die in die Vorauswahl aufgenommen, auf Eignung geprüft und in die Übersicht eingeschlossen wurden, mit Begründung für Ausschluss in jeder Stufe, idealerweise unter Verwendung eines Flussdiagramms.

Studienmerkmale

Risiko der Verzerrung innerhalb der Studien

Ergebnisse der

einzelnen Studien

Ergebnissynthese

Risiko von Verzerrungen

über Studien hinweg

Zusätzliche Analysen

19 Daten zum Risiko von Verzerrungen innerhalb jeder Studie und, falls verfügbar, eine Beurteilung der Güte der Zielkriterien (siehe Item 12).

20 Für jede Studie Darstellung aller Endpunkte (Wirksamkeit und Nebenwirkungen): (a) einfache zusammenfassende Daten für jede Interventionsgruppe, (b) Effektschätzer und Konfidenzintervalle, idealerweise mit Forest Plot.

21 Darstellung der Meta-Analyse, einschließlich Konfidenzintervalle und Heterogenitätsmaße.

22 Darstellung der Ergebnisse zur Beurteilung des Risikos von Verzerrungen über alle Studien hinweg (siehe Item 15).

23 Präsentation der Ergebnisse der zusätzlichen Analysen, falls durchgeführt (z. B. Sensitivitäts- oder Subgruppenanalysen, Meta-Regression [siehe Item 16]).

Diskussion

Zusammenfassung der 24 Evidenz Einschränkungen

Zusammenfassung der Hauptergebnisse einschließlich der Stärke der Evidenz für jedes Hauptzielkriterium; Relevanz für Zielgruppen (z.B. Gesundheitsdienstleister, Anwender, politische Entscheidungsträger).

Schlussfolgerungen

Diskutiere Einschränkungen der Studie auf Studienebene und auf Ebene der Zielkriterien (z.B. Risiko von Verzerrungen) sowie auf Ebene der Übersicht (z.B. unvollständiges Auffinden der identifizierten Forschung, Verzerrung des Berichts). Interpretation der Ergebnisse unter Berücksichtigung des Stands der Forschung und Schlussfolgerungen für weitere Forschung.

Finanzielle Unterstützung

Funktion der Geldgeber für die systematische Übersicht. 


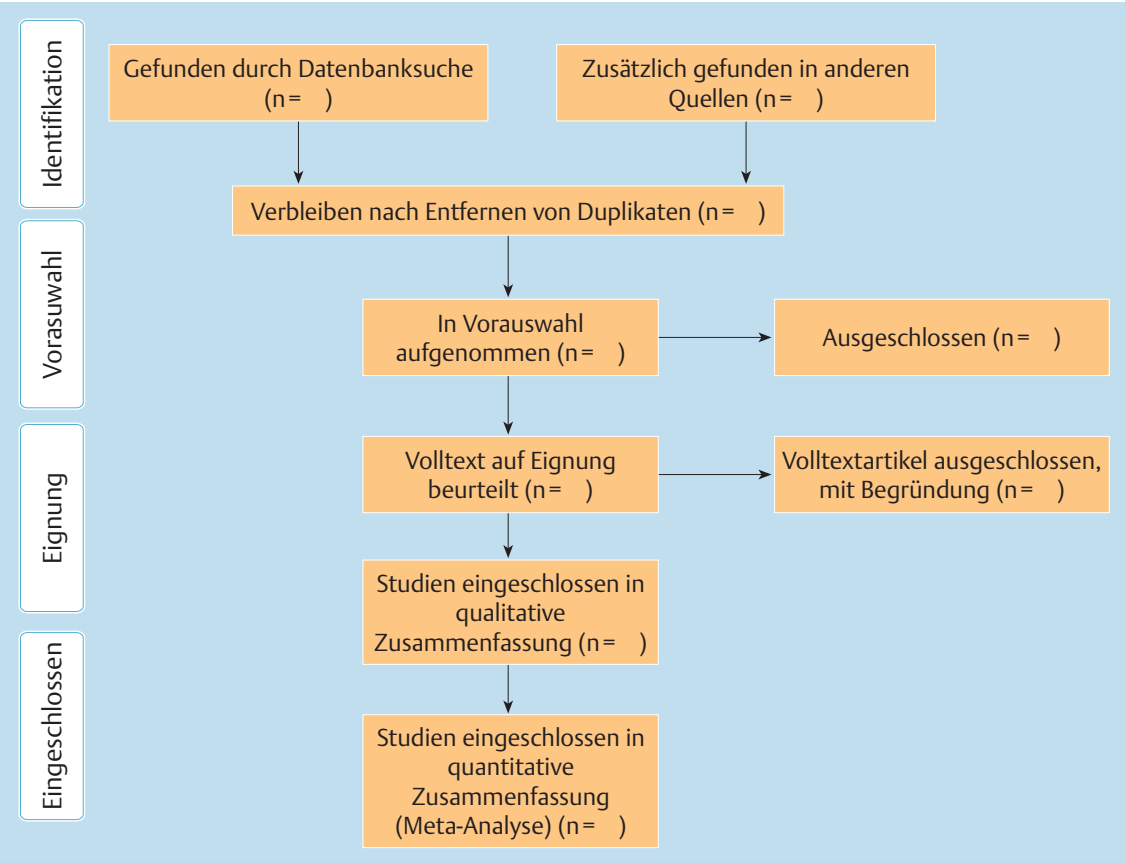

Abb. 1 Flussdiagramm zur Beschreibung der verschiedenen Phasen einer systematischen Übersicht (herunterladbare Vorlage, die von Wissenschaftlern verwendet werden kann).

anderer Arbeiten, die möglicherweise für das Treffen relevant sein könnten, insbesondere im Hinblick auf die Veränderung der Checkliste. Es wurde eine internationale Befragung unter Autoren von Übersichten, Endnutzern und Gruppen, die systematische Übersichten und MetaAnalysen beauftragen oder nutzen, durchgeführt. Hierzu gehörten das „International Network of Agencies for Health Technology Assessment“ (INAHTA) und das „Guidelines International Network“ (GIN). Ziel dieser Befragung war, Meinungen zum QUOROMStatement zu erhalten, inklusive der Vorzüge der existierenden Checkliste. Die Ergebnisse dieser Vorarbeiten wurden während des Treffens präsentiert; sie sind auf der PRISMA-Webseite zusammengefasst

(http://www.prisma-statement.org/).

Nur Punkte, die als wesentlich eingeschätzt wurden, wurden auf der Checkliste beibehalten oder neu hinzugefügt. Einige zusätzliche Punkte sind nichtsdestotrotz wünschenswert, und Autoren von Übersichten sollten diese, wenn relevant, einschließen [35]. So ist es z.B. hilfreich anzugeben, ob eine systematische Übersicht eine Aktualisierung einer früheren oder eine neue Zusammenfassung ist [25]. Außerdem sollten bei Aktualisierungen Änderungen im Vorgehen im Vergleich zum ursprünglichen Reviewprotokoll beschrieben werden.
Zeitnah wurde nach dem Treffen ein Entwurf der PRISMA-Checkliste in der Gruppe zirkuliert, der sowohl die Tagungsteilnehmer einschloss als auch diejenigen, die eingeladen, aber verhindert waren. Eine Datei wurde erstellt, in der die Kommentare und Überarbeitungen jeder Person vermerkt wurden. Daraufhin wurde die Checkliste in 11 Punkten verändert. Die Gruppe stimmte der Checkliste, dem Flussdiagramm und der englischsprachigen Fassung dieser Publikation zu.

Obwohl keine direkte Evidenz dafür gefunden wurde, dass einige Punkte verbleiben oder ergänzt werden sollten, wurde die Evidenz anderer Themengebiete als relevant eingeschätzt. So werden die Autoren in Punkt 5 gebeten, wenn möglich Informationen zur Registrierung der systematischen Übersicht einschließlich Registrierungsnummer anzugeben. Obwohl die Registrierung systematischer Übersichten bisher noch nicht weit verfügbar ist $[38,39]$, verlangen inzwischen alle Zeitschriften, die dem „International Committee of Medical Journal Editors“ (ICMJE) angehören [11], die Registrierung aller klinischer Studien, um Transparenz und Verantwortlichkeit zu erhöhen [41]. Diese Vorteile werden wahrscheinlich auch denen zugutekommen, die systematische Übersichten erstellen, indem möglicherweise dem Risiko einer großen Anzahl von Übersichten zu identischen Fragestellun- gen vorgebeugt wird $[3,5]$ und größere Transparenz bei der Aktualisierung einer systematischen Übersicht erreicht wird.

\section{Das PRISMA-Statement \\ $\nabla$}

Das PRISMA-Statement besteht aus einer 27 Punkte Checkliste (Tab. 1) und einem Flussdiagramm, das in vier Phasen aufgeteilt ist ( Abb. 1).

Das PRISMA-Statement soll Autoren dabei unterstützen, das Berichten von systematischen Übersichten und Meta-Analysen zu verbessern. Wir haben den Schwerpunkt auf randomisierte Studien gelegt, doch kann PRISMA auch als Grundlage für das Berichten von systematischen Übersichten anderer Studien, insbesondere Interventionsstudien verwendet werden. PRISMA kann bei der kritischen Bewertung publizierter systematischer Übersichten ebenfalls hilfreich sein. Allerdings ist PRISMA kein Instrument zur Abschätzung der Qualität einer systematischen Übersicht.

\section{Von QUOROM zu PRISMA} $\nabla$

Die neue PRISMA-Checkliste unterscheidet sich in verschiedenen Punkten von der QUOROM-Checkliste, und die wesentlichen Änderungen werden in Tab. 2 hervorgehoben.

Allgemein entkoppelt die PRISMA Checkliste einige Punkte aus der QUOROM Checkliste und verschiedene Punkte der Checkliste werden, wo sinnvoll, miteinander verbunden, um die Konsistenz in der systematischen Übersicht zu verbessern.

Auch das Flussdiagramm hat Veränderungen erfahren. Vor Studieneinschluss und der Aufzählung von Gründen für den Ausschluss anderer Studien muss zuerst die Literaturrecherche stehen. Diese Suche ergibt eine Liste von Treffern, die zunächst auf Einschlusskriterien hin überprüft werden. Entsprechend wird sich die Zahl der verbleibenden Artikel reduzieren. Die Anzahl der eingeschlossenen Artikel kann kleiner oder größer als die Anzahl der Studien sein, weil ein Artikel über mehrere Studien berichten kann und weil die Ergebnisse einer Studie in mehreren Artikeln beschrieben werden können. Um dies zu klären, sollen im 
Tab. 2 Wesentliche spezifische Änderungen zwischen der QUOROM-Checkliste und der PRISMA-Checkliste. Ein Plus zeigt das Vorhandensein in QUOROM oder PRISMA an.

\begin{tabular}{|c|c|c|c|c|}
\hline $\begin{array}{l}\text { Abschnitt/ } \\
\text { Thema }\end{array}$ & IPunkt & QUOROM & PRISMA & Kommentar \\
\hline $\begin{array}{l}\text { Zusammen- } \\
\text { fassung }\end{array}$ & & $x$ & $x$ & $\begin{array}{l}\text { Sowohl QUOROM als auch PRISMA fordern eine Zusammenfassung. PRISMA gibt } \\
\text { kein spezifisches Format vor. }\end{array}$ \\
\hline Einleitung & Ziele & & $x$ & $\begin{array}{l}\text { Dieser neue Punkt (4) adressiert die Fragestellung. Diese soll unter Verwendung des } \\
\text { PICO Berichts-Systems formuliert werden (Teilnehmer, Interventionen, Vergleiche, } \\
\text { Zielkriterien; engl.: participants, interventions, comparisons, outcome(s)). Zusammen } \\
\text { mit dem spezifischen Studiendesign ergibt sich PICOS; dieser Punkt ist verknüpft mit } \\
\text { den Punkten 6, } 11 \text { und } 18 \text { der Checkliste. }\end{array}$ \\
\hline Methoden & Protokoll & & $x$ & $\begin{array}{l}\text { Dieser neue Punkt (5) fragt ab, ob der Übersicht ein Protokoll zugrundeliegt und, falls } \\
\text { vorhanden, wie auf dieses zugegriffen werden kann. }\end{array}$ \\
\hline Methoden & Suche & $x$ & $x$ & $\begin{array}{l}\text { Obwohl das Berichten der Suche in der QUOROM- und der PRISMA-Checkliste } \\
\text { vorhanden ist, fordert PRISMA die vollständige Beschreibung mindestens einer } \\
\text { elektronischen Suchstrategie (Item 8). Ohne diese Beschreibung ist es unmöglich, } \\
\text { die von den Autoren durchgeführte Suche zu replizieren. }\end{array}$ \\
\hline Methoden & $\begin{array}{l}\text { Beurteilung des Risikos } \\
\text { von Verzerrungen in den } \\
\text { eingeschlossenen Studien }\end{array}$ & $x$ & $x$ & $\begin{array}{l}\text { Umbenannt von „Qualitätsbeurteilung“ in QUOROM. Dieser Punkt (12) ist verknüpft } \\
\text { mit dem Berichten dieser Information in den Ergebnissen (Punkt 19). Es wurde das } \\
\text { neue Konzept der Beurteilung auf Zielkriterien-Ebene eingeführt. }\end{array}$ \\
\hline Methoden & $\begin{array}{l}\text { Beurteilung des Risikos } \\
\text { von Verzerrungen über } \\
\text { die Studien hinweg }\end{array}$ & & $x$ & $\begin{array}{l}\text { Dieser neue Punkt (15) fordert die Autoren auf, jegliche Beurteilung des Risikos von } \\
\text { Verzerrungen in der Übersicht zu beschreiben. Hierzu gehört das selektive Berichten } \\
\text { innerhalb der eingeschlossenen Studien. Dieser Punkt ist verknüpft mit dem Berichten } \\
\text { dieser Informationen in den Ergebnissen (Item 22). }\end{array}$ \\
\hline Diskussion & & $x$ & $x$ & $\begin{array}{l}\text { Obwohl sowohl die QUOROM- als auch die PRISMA-Checkliste den Abschnitt Diskussi- } \\
\text { on adressieren, widmet PRISMA drei Punkte (24-26) der Diskussion. In PRISMA wer- } \\
\text { den die Hauptgründe für Einschränkungen einer Studie explizit genannt, und es ist eine } \\
\text { Diskussion dieser Einschränkungen erforderlich. }\end{array}$ \\
\hline $\begin{array}{l}\text { Finanzielle } \\
\text { Unterstützung }\end{array}$ & & & $x$ & $\begin{array}{l}\text { Dieser neue Punkt (27) fordert die Autoren auf, Informationen zur finanziellen } \\
\text { Unterstützung jedweder Art der systematischen Übersicht darzulegen. }\end{array}$ \\
\hline
\end{tabular}

PRISMA-Flussdiagramm nun die Informationen aus den verschiedenen Phasen des Reviewprozesses dargestellt werden.

\section{Befürwortung \\ $\checkmark$}

Zeitschriften, die das QUOROM-Statement befürwortet haben, sollten dieses durch das PRISMA-Statement ersetzen. Wir hoffen, dass andere Zeitschriften das PRISMA-Statement unterstützen, was durch eine Registrierung auf der PRISMA-Webseite geschehen kann. Um die Wichtigkeit eines transparenten Berichts einer systematischen Übersicht zu betonen, bitten wir die Zeitschriften, die das PRISMA-Statement unterstützen, auf PRISMA zu verweisen und die Webseite des PRISMA-Statements in die Hinweise für Autoren aufzunehmen. Wir laden außerdem Herausgeber dazu ein, PRISMA $\mathrm{zu}$ befürworten und Autoren zu ermutigen, die Prinzipien im PRISMA-Statement einzuhalten.

\section{Das PRISMA-Explanation und Elaboration Paper $\checkmark$}

Zusätzlich zum PRISMA-Statement wurde eine Arbeit zur Erklärung und ausführlichen Darstellung („explanation and elaboration paper") erstellt [22], die im Stil den Erklärungen anderer Empfehlungen folgt $[1,6,40]$. Der Prozess, der zu diesem Dokument führte, schloss die Entwicklung einer großen Datenbank mit Musterbeispielen ein, um zu illustrieren, wie jeder Punkt der Checkliste am besten berichtet werden sollte. Es wurde ebenfalls eine umfassende Evidenzbasis gesammelt, um jeden Punkt in der Checkliste zu begründen. Das Erklärungsdokument wurde nach mehreren persönlichen Treffen und zahlreichen Wiederholungsschritten zwischen einigen Teilnehmern des Treffens vervollständigt. Danach erhielt es die gesamte Gruppe zur zusätzlichen Revision und endgültigen Zustimmung. Abschließend bildete die Gruppe ein Disseminations-Komitee, das die Verbreitung und Implementierung von PRISMA unterstützen soll.

\section{Diskussion}

$\checkmark$

Die Qualität des Berichtens systematischer Übersichten ist immer noch nicht optimal $[4,10,13,18,29]$. In einer kürzlich veröffentlichten Übersicht von 300 systematischen Übersichtsarbeiten wurde berichtet, dass nur wenige Autoren eine mögliche Publikationsverzerrung betrachteten [24], obwohl die Beweislage für ihre Existenz [14] und ihr Einfluss auf die Ergebnisse systematischer Übersichten erdrückend sind [36]. Selbst wenn die Möglichkeit der Publikationsverzerrung beurteilt wird, ist dies keine Garantie, dass die Autoren der Übersichten diese adäquat eingeschätzt und beurteilt haben [20]. Obwohl das Fehlen des Berichts einer solchen Untersuchung nicht notwendigerweise darauf hinweist, dass diese nicht durchgeführt wurde, ist die Angabe, dass Publikationsverzerrung untersucht wurde, vermutlich ein Indikator für eine gründliche Durchführung der systematischen Übersicht.

Es wurden mehrere Ansätze entwickelt, um systematische Übersichten bei einer größeren Themenvielfalt durchzuführen. 
So werden jetzt z.B. systematische Übersichten zur Untersuchung von Wirtschaftlichkeit [19], von diagnostischen [12] oder prognostischen Fragestellungen [2], von genetischen Assoziationen [17] und der Entwicklung von Entscheidungsgrundlagen und -richtlinien [21] erstellt. Die allgemeinen Konzepte und Themen, die durch PRISMA abgedeckt werden, sind für jede systematische Übersicht relevant, nicht nur für Fragen zu Wirkung und Nebenwirkungen von Interventionen im Bereich der medizinischen Versorgung. Allerdings werden bestimmte Situationen Modifikationen von Punkten der Checkliste oder des Flussdiagramms erfordern. So ist beispielsweise die Beurteilung des Risikos von Verzerrungen ein zentrales Konzept, aber die Punkte, die dafür in systematischen Übersichten von Diagnosestudien verwendet werden, zielen vermutlich auf Aspekte wie das Spektrum von Patienten und die Verifizierung des Krankheitsstatus ab und diese Schwerpunkte unterscheiden sich von Übersichten für Interventionen. Das Flussdiagramm wird ebenfalls modifiziert werden müssen, wenn individuelle Patientendaten in Meta-Analysen verwendet werden [34].

Wir haben ein Dokument zur näheren Erklärung erstellt [22], um die Nützlichkeit von PRISMA zu erhöhen. Für jeden Punkt der Checkliste enthält dieses Dokument ein Musterbeispiel für gutes Berichten, die Rationale für die Aufnahme in der Checkliste und zusätzliche Evidenz einschließlich Referenzen - wenn möglich. Wir sind davon überzeugt, dass dieses Dokument beim Unterrichten der Methodologie systematischer Übersichtsarbeiten ebenfalls eine nützliche Informationsquelle ist. Wir bitten Zeitschriften, in ihren Hinweisen für Autoren das erklärende Dokument mit aufzunehmen.

PRISMA ist ein lebendes Dokument, wie alle Bemühungen um evidenzbasiertes Vorgehen. Daher laden wir Leser ein, die überarbeitete Fassung des Statements, insbesondere die neue Checkliste und das Flussdiagramm auf der PRISMAWebseite, zu kommentieren. Diese Hinweise werden die Grundlage für die stetige Weiterentwicklung von PRISMA bilden.

Danksagung: Folgende Personen trugen zum PRISMA Statement bei: Doug Altman, DSc, Centre for Statistics in Medici-

\section{Zusätzliche Informationen}

Abb. 1 Flussdiagramm zur Beschreibung der verschiedenen Phasen einer systematischen Übersicht (herunterladbare Vorlage, die von Wissenschaftlern verwendet werden kann)

Englisches Original verfügbar unter: DOI:10.1371/journal.pmed.1000097.s001

Deutsche Übersetzung verfügbar unter: http://dx.doi.org/10.1055/s-0031-

1272978

Tab.1 Checkliste der Items für den Bericht einer systematischen Übersicht oder Meta-Analyse (herunterladbare Vorlage, die von Wissenschaftlern verwendet werden kann)

Englisches Original verfügbar unter: DOI:10.1371/journal.pmed.1000097.s002 Deutsche Übersetzung verfügbar unter: http://dx.doi.org/10.1055/s-00311272978

ne (Oxford, Großbritannien); Gerd Antes, $\mathrm{PhD}$, Universitätsklinikum Freiburg (Freiburg, Deutschland); David Atkins, MD, MPH, Health Services Research and Development Service, Veterans Health Administration (Washington, D. C., Vereinigte Staaten von Amerika); Virginia Barbour, MRCP, DPhil, PLoS Medicine (Cambridge, Großbritannien); Nick Barrowman, PhD, Children's Hospital of Eastern Ontario (Ottawa, Kanada); Jesse A. Berlin, ScD, Johnson \& Johnson Pharmaceutical Research and Development (Titusville, New Jersey, Vereinigte Staaten von Amerika); Jocalyn Clark, PhD, PLoS Medicine (zur Zeit des Schreibens, BMJ, London, Großbritannien); Mike Clarke, PhD, UK Cochrane Centre (Oxford, Großbritannien) und School of Nursing and Midwifery, Trinity College (Dublin, Irland); Deborah Cook, MD, Departments of Medicine, Clinical Epidemiology and Biostatistics, McMaster University (Hamilton, Kanada); Roberto D'Amico, PhD, Universitá di Modena e Reggio Emilia (Modena, Italien) and Centro Cochrane Italiano, Istituto Ricerche Farmacologiche Mario Negri (Mailand, Italien); Jonathan J. Deeks, PhD, University of Birmingham (Birmingham, Großbritannien); P. J. Devereaux, MD, PhD, Departments of Medicine, Clinical Epidemiology and Biostatistics, McMaster University (Hamilton, Kanada); Kay Dickersin, PhD, Johns Hopkins Bloomberg School of Public Health (Baltimore, Maryland, Vereinigte Staaten von Amerika); Matthias Egger, MD, Department of Social and Preventive Medi- cine, Universität Bern (Bern, Schweiz); Edzard Ernst, MD, PhD, FRCP, FRCP(Edin), Peninsula Medical School (Exeter, Großbritannien); Peter C. Gøtzsche, MD, MSc, The Nordic Cochrane Centre (Kopenhagen, Dänemark); Jeremy Grimshaw, MBChB, PhD, FRCFP, Ottawa Hospital Research Institute (Ottawa, Kanada); Gordon Guyatt, MD, Departments of Medicine, Clinical Epidemiology and Biostatistics, McMaster University (Hamilton, Kanada); Julian Higgins, PhD, MRC Biostatistics Unit (Cambridge, Großbritannien); John P. A. Ioannidis, MD, University of Ioannina Campus (Ioannina, Griechenland); Jos Kleijnen, MD, PhD, Kleijnen Systematic Reviews Ltd. (York, Großbritannien) und School for Public Health and Primary Care (CAPHRI), University of Maastricht (Maastricht, Niederlande); Tom Lang, MA, Tom Lang Communications and Training (Davis, California, Vereinigte Staaten von Amerika); Alessandro Liberati, MD, Università di Modena e Reggio Emilia (Modena, Italien) und Centro Cochrane Italiano, Istituto Ricerche Farmacologiche Mario Negri (Mailand, Italien); Nicola Magrini, MD, NHS Centre for the Evaluation of the Effectiveness of Health Care - CeVEAS (Modena, Italien); David McNamee, PhD, The Lancet (London, Großbritannien); Lorenzo Moja, MD, MSc, Centro Cochrane Italiano, Istituto Ricerche Farmacologiche Mario Negri (Mailand, Italien); David Moher, PhD, Ottawa Methods Centre, Ottawa Hospital Research Institute (Ottawa, Kanada); Cynthia Mulrow, MD, MSc, Annals of Internal Medicine (Philadelphia, Pennsylvania, Vereinigte Staaten von Amerika); Maryann Napoli, Center for Medical Consumers (New York, New York, Vereinigte Staaten von Amerika); Andy Oxman, MD, Norwegian Health Services Research Centre (Oslo, Norwegen); Ba' Pham, MMath, Toronto Health Economics and Technology Assessment Collaborative (Toronto, Kanada) (beim ersten Treffen der Gruppe, GlaxoSmithKline Kanada, Mississauga, Kanada); Drummond Rennie, MD, FRCP, FACP, University of California San Francisco (San Francisco, Kalifornien, Vereinigte Staaten von Amerika); Margaret Sampson, MLIS, Children's Hospital of Eastern Ontario (Ottawa, Kanada); Kenneth F. Schulz, PhD, MBA, Family Health International (Durham, North Carolina, Vereinigte Staaten von Amerika); Paul G. Shekelle, $\mathrm{MD}, \mathrm{PhD}$, Southern California Evidence Based Practice Center (Santa Monica, Kalifornien, Vereinigte Staaten von Ameri- 
Konzeptionelle Aspekte bei der Weiterentwicklung von QUOROM zu PRISMA

\section{Erstellen einer systematischen Übersicht ist ein iterativer Prozess}

Die Durchführung einer systematischen Übersicht hängt sehr von dem Ziel und der Qualität der eingeschlossenen Studien ab. Daher ist möglicherweise eine Änderung des ursprünglichen Studienprotokolls für die systematische Übersicht während ihrer Durchführung erforderlich. Ein Leitfaden zum Berichten systematischer Übersichten sollte die Empfehlung aussprechen, dass derartige Änderungen berichtet und begründet werden können, ohne dass diese als inadäquat eingestuft werden. Das PRISMAStatement (Punkte 5, 11, 16 und 23) trägt diesem iterativen Prozess Rechnung. Während alle Cochrane Reviews auf einem Studienprotokoll basieren sollten, wird nur in etwa $10 \%$ der systematischen Übersichten berichtet, dass sie nach einem Studienprotokoll arbeiten [24]. Ohne ein frei zugängliches Studienprotokoll ist allerdings die Unterscheidung adäquater und inadäquater Änderungen schwierig.

Forschung Durchführen und Berichten sind verschiedene Konzepte

Diese Unterscheidung ist für systematische Übersichten weniger offensichtlich als für die Beurteilung des Berichts einer einzelnen Studie, weil die Durchführung und das Berichten von systematischen Übersichten von Natur aus eng miteinander verbunden sind. Wird beispielsweise in einer systematischen Übersicht die Beurteilung des Risikos von Verzerrungen bei den eingeschlossenen Studien nicht berichtet, kann dieses als Kennzeichen geringer Durchführungsqualität gedeutet werden, weil die Beurteilung dieses Risikos für systematische Übersichten besonders relevant ist [26].

Beurteilung des Verzerrungsrisikos auf der Ebene einzelner Studien im Vergleich zur Schlussfolgerung

Studien, die in einer systematischen Übersicht Berücksichtigung finden, müssen sorgfältig hinsichtlich des Risikos von Verzerrungen auf zwei Ebenen überprüft werden. Das ist zum einen die Beurteilung auf der Studien-Ebene (z. B.: ist die Zuteilung der Behandlung geheim gehalten worden) und für einige Merkmale zum anderen die Beurteilung auf der Schlussfolgerungs-Ebene. Letztere schließt die Untersuchung von Reliabilität und Validität der Daten für jeden relevanten Endpunkt ein. Hier müssen die Verfahren bestimmt werden, mit denen die Endpunkte in jeder einzelnen Studie erhoben werden [16]. Die Güte der Evidenz kann selbst innerhalb einer Studie variieren, z.B. zwischen der Erfassung eines primären Endpunkts, der wahrscheinlich sehr sorgfältig und systematisch gemessen wird, und der Erhebung von schweren unerwünschten Ereignissen [32], die möglicherweise von spontanen Berichten der Untersucher abhängen. Diese Information sollte berichtet werden, um eine explizite Beurteilung zu erlauben, inwieweit der Effektschätzer korrekt ist [32].

\section{Bedeutung von Verzerrungen beim Berichten}

Verschiedene Arten von Verzerrungen beim Berichten behindern möglicherweise die Durchführung und die Interpretation systematischer Übersichten. Selektives Berichten vollständiger Studien (d.h. Publikationsverzerrung) [14] sowie die erst kürzlich empirisch gezeigte Ergebnisberichtsverzerrung innerhalb einzelner Studie [8, 9] sollte von Wissenschaftlern bei der Durchführung und dem Berichten einer systematischen Übersicht berücksichtigt werden. Obwohl die Auswirkungen dieser Verzerrungen für die Durchführung und das Berichten systematischer Übersichten per se unklar sind, ist selektives Berichten von Ergebnissen auch bei systematischen Übersichten zu finden [33].

ka); Jennifer Tetzlaff, BSc, Ottawa Methods Centre, Ottawa Hospital Research Institute (Ottawa, Kanada); David Tovey, FRCGP, The Cochrane Library, Cochrane Collaboration (Oxford, Großbritannien) (at the time of the first meeting of the group, BMJ, London, Großbritannien); Peter Tugwell, MD, MSc, FRCPC, Institute of Population Health, University of Ottawa (Ottawa, Kanada).

Finanzielle Unterstützung: PRISMA wurde finanziell unterstützt durch die Canadian Institutes of Health Research, die lehrstuhl der Universität von Ottawa.
Keiner der Förderer war in die Planung, Durchführung sowie das Schreiben der PRISMA Dokumente involviert. Weiterhin hatte keiner der Sponsoren eine Aufgabe beim Entwerfen des Manuskripts.

Beiträge der Autoren: Die ICMJE Kriterien für Autorenschaften wurden zur Kenntnis genommen und erfüllt von DM, AL, JT und DGA. Schrieben den ersten Entwurf des Artikels: DM, AL, DGA. Trugen zum Schreiben des Artikels bei: DM, AL, JT, DGA. Nahmen an regelmäßig stattfindenden Telefonkonferenzen teil, identifizierten die Teilnehmer, sicherten die finanzielle Unterstützung, planten das Treffen, nahmen an dem Treffen teil und entwarfen das Manuskript: DM, AL, DGA. Nahm an der Identifikation der Evidenz für PRISMA teil, verbesserte die Checkliste und entwarf das Manuskript: JT. Stimmen den Empfehlungen zu: DM, AL, JT, DGA.

[Veröffentlichung des Originals: PLoS Medicine 2009; 6:e1000097; doi:10.1371/journal.pmed.1000097]

Literatur

1 Altman DG, Schulz KF, Moher D et al. The revised CONSORT statement for reporting randomized trials: explanation and elaboration. Ann Intern Med 2001; 134: 663694

2 Altman DG. Systematic reviews of evaluations of prognostic variables. BMJ 2001; 323: 224-228

3 Bagshaw SM, McAlister FA, Manns BJ, Ghali $W A$. Acetylcysteine in the prevention of contrast-induced nephropathy: a case study of the pitfalls in the evolution of evidence. Arch Intern Med 2006; 166: 161166

4 Bhandari M, Morrow F, Kulkarni AV, Tornetta 3rd $P$. Meta-analyses in orthopaedic surgery. A systematic review of their methodologies. J Bone Joint Surg Am 2001; 83-A: $15-24$

5 Biondi-Zoccai GG, Lotrionte M, Abbate A et al. Compliance with QUOROM and quality of reporting of overlapping meta-analyses on the role of acetylcysteine in the prevention of contrast associated nephropathy: case study. BMJ 2006; 332: 202-209

6 Bossuyt PM, Reitsma JB, Bruns DE et al. Towards complete and accurate reporting of studies of diagnostic accuracy: The STARD Initiative. Ann Intern Med 2003; 138: 4044

enischen Ministeriums der Universität unterstützt (COFIN - PRIN 2002 prot. 2002061749 and COFIN - PRIN 2006 prot. 2006062298). DGA wird durch Cancer Research UK unterstützt. DM wird gefördert durch einen Forschungs-

7 Canadian Institutes of Health Research. Randomized controlled trials registration/ application checklist (12/2006) 2006; http://www.cihr-irsc.gc.ca/e/documents/ rct_reg_e.pdf [zugegriffen am 5.7.2010] 
8 Chan AW, Hrobjartsson A, Haahr MT, Gotzsche PC, Altman DG. Empirical evidence for selective reporting of outcomes in randomized trials: comparison of protocols to published articles. JAMA 2004; 291: 24572465

9 Chan AW, Krleza-Jeric K, Schmid I, Altman $D G$. Outcome reporting bias in randomized trials funded by the Canadian Institutes of Health Research. CMAJ 2004; 171: 735740

10 Choi PT, Halpern SH, Malik $N$ et al. Examining the evidence in anesthesia literature: a critical appraisal of systematic reviews. Anesth Analg 2001; 92: 700-709

11 De Angelis $C D$, Drazen JM, Frizelle FA et al. Is this clinical trial fully registered? A statement from the International Committee of Medical Journal Editors. CMAJ 2005; 172: 1700-1702

12 Deeks JJ. Systematic reviews in health care: Systematic reviews of evaluations of diagnostic and screening tests. BMJ 2001; 323: 157-162

13 Delaney A, Bagshaw SM, Ferland A et al. A systematic evaluation of the quality of meta-analyses in the critical care literature. Crit Care 2005; 9: R575-58

14 Dickersin K. Publication bias: Recognizing the problem, understanding its origins and scope, and preventing harm.In: Rothstein HR, Sutton AJ, Borenstein M eds (Hrsg). Publication bias in meta-analysis-Prevention, assessment and adjustments. Chichester (UK): John Wiley \& Sons, 2005: 11-33

15 Green S, Higgins J. Glossary. Cochrane handbook for systematic reviews of interventions 4.2.5. The Cochrane Collaboration. 2005; http://www.cochrane.org/resources/glossary.htm [zugegriffen am 4.7.2010]

16 Guyatt GH, Oxman AD, Vist GE et al. GRA$\mathrm{DE}$ : an emerging consensus on rating quality of evidence and strength of recommendations. BMJ 2008; 336: 924-926

17 Ioannidis JP, Ntzani EE, Trikalinos TA, Contopoulos-Ioannidis DG. Replication validity of genetic association studies. Nat Genet 2001; 29: 306-309

18 Kelly $K D$, Travers $A$, Dorgan $M$, Slater $L$ Rowe $\mathrm{BH}$. Evaluating the quality of systematic reviews in the emergency medicine literature. Ann Emerg Med 2001; 38: 518 526

19 Ladabaum U, Chopra CL, Huang G et al. Aspirin as an adjunct to screening for prevention of sporadic colorectal cancer. A cost-effectiveness analysis. Ann Intern Med 2001; 135: 769-781

20 Lau J, Ioannidis JP, Terrin N, Schmid CH, Olkin $I$. The case of the misleading funnel plot. BMJ 2006; 333: 597-600

21 Lavis J, Davies H, Oxman A et al. Towards systematic reviews that inform health care management and policy-making. J Health Serv Res Policy 2005; 10 Suppl 1: 35-48

22 Liberati A, Altman DG, Tetzlaff J et al. The PRISMA statement for reporting systematic reviews and meta-analyses of stu- dies that evaluate health care interventions: explanation and elaboration. PLoS Med 2009; 6: e1000100

23 Moher D, Cook DJ, Eastwood S et al. Improving the quality of reports of meta-analyses of randomised controlled trials: the QUOROM statement. Quality of Reporting of Meta-analyses. Lancet 1999; 354: 18961900

24 Moher D, Tetzlaff J, Tricco AC, Sampson M, Altman DG. Epidemiology and reporting characteristics of systematic reviews. PLoS Med 2007; 4: e78

25 Moher D, Tsertsvadze A. Systematic reviews: when is an update an update? Lancet 2006; 367: 881-883

26 Moja LP, Telaro E, D’Amico $R$ et al. Assessment of methodological quality of primary studies by systematic reviews: results of the metaquality cross sectional study. BM] 2005; 330: 1053

27 Mulrow CD. The medical review article: state of the science. Ann Intern Med 1987; 106: $485-488$

28 Oxman $A D$, Cook DJ, Guyatt GH. Users' guides to the medical literature. VI. How to use an overview. Evidence-Based Medicine Working Group. JAMA 1994; 272: 13671371

29 Richards $D$. The quality of systematic reviews in dentistry. Evid Based Dent 2004; 5: 17

30 Sacks HS, Berrier J, Reitman D, Ancona-Berk VA, Chalmers TC. Meta-analyses of randomized controlled trials. N Engl J Med 1987; 316: 450-455

31 Sacks HS, Reitman D, Pagano D, Kupelnick $B$. Meta-analysis: an update. Mt Sinai J Med 1996; 63: 216-224

32 Schünemann HJ, Jaeschke R, Cook DJ et al. An official ATS statement: grading the quality of evidence and strength of recommendations in ATS guidelines and recommendations. Am J Respir Crit Care Med 2006; 174: 605-614

33 Silagy CA, Middleton P, Hopewell S. Publishing protocols of systematic reviews: comparing what was done to what was planned. JAMA 2002; 287: 2831-2834

34 Stewart LA, Clarke MJ. Practical methodology of meta-analyses (overviews) using updated individual patient data. Cochrane Working Group. Stat Med 1995; 14: 2057 2079

35 Strech D, Tilburt J. Value judgments in the analysis and synthesis of evidence. J Clin Epidemiol 2008; 61: 521-524

36 Sutton AJ. Evidence concerning the consequences of publication and related biases.In: Rothstein HR, Sutton AJ, Borenstein $\mathrm{M}$ eds (Hrsg). Publication bias in metaanalysis-Prevention, assessment and adjustments. Chichester (UK): John Wiley \& Sons, 2005: 175-192

37 Swingler GH, Volmink J, Ioannidis JP. Number of published systematic reviews and global burden of disease: database analysis. BMJ 2003; 327: 1083-1084
38 The Joanna Briggs Institute. Protocols \& work in progress 2008; http://www.joannabriggs.edu.au/pubs/

systematic_reviews_prot.php [zugegriffen am 04.07.2010]

39 University of York. Centre for Reviews and Dissemination. 2009; http:/ www.york.ac.uk/inst/crd/ [zugegriffen am 4.7.2010]

40 Vandenbroucke JP, von Elm E, Altman DG et al. Strengthening the Reporting of Observational Studies in Epidemiology (STRO$\mathrm{BE})$ : explanation and elaboration. Ann Intern Med 2007; 147: W163-194

41 Whittington $C J$, Kendall T, Fonagy $P$ et al. Selective serotonin reuptake inhibitors in childhood depression: systematic review of published versus unpublished data. Lancet 2004; 363: 1341-1345

42 Young $C$, Horton $R$. Putting clinical trials into context. Lancet 2005; 366: 107-108 